\title{
« Tout est bon dans le phoque » : réimaginer l'ailleurs contemporain avec Jean Echenoz
}

\author{
Sara-Bédart Goulet \\ Figura, UQAM/LLA-CREATIS, Toulouse
}

L'époque contemporaine, qualifiée de surmoderne par l'anthropologue Marc Augé, serait caractérisée par un excès d'espace, « corrélatif du rétrécissement de la planète » (44). En effet, la banalisation et l'accélération des moyens de transports et de diffusion de l'information donnent lieu à de considérables changements d'échelle du monde tel qu'il nous apparaît. Les images qui peuplent notre quotidien, qu'elles soient documentaires ou fictives, rendent familiers certains lieux éloignés, que nous reconnaissons, souligne Augé, même si nous ne les connaissons pas (45). Ce contexte de surabondance spatiale suggère que les représentations de l'ailleurs souffrent d'un imaginaire asséché et figé, notamment par le flot d'images qui circule des coins les plus reculés de la planète, autrefois associés à un lointain fantasmatique. En effet, si, comme l'affirmait Victor Segalen, la sensation d'exotisme s'émousse rapidement une fois la surprise passée (33), l'accessibilité directe ou indirecte à l'ailleurs et l'adaptabilité croissante des humains à un monde en rapide transformation pourraient amenuiser la différence sur laquelle l'écrivain fait reposer son Essai sur l'exotisme (41) et, dès lors, affaiblir le dépaysement que pouvaient autrefois offrir des récits décrivant des pays étrangers au lecteur. D'autant que le divers géographique, le plus connu parmi les manifestations de l'exotisme, serait davantage menacé par la dégradation et cela, dès le début du XX ${ }^{\text {ème }}$ siècle, au moment où Segalen déplore les effets néfastes du tourisme et l'attitude qu'il engendre vis-à-vis de l'ailleurs (71).

L'œuvre romanesque de l'auteur français Jean Echenoz (né en 1947), qu'il qualifie lui-même de " géographique » (Harang), et dont les personnages semblent être d'éternels voyageurs, forme un terrain fertile pour penser l'ailleurs et sa signification à notre époque par le biais du littéraire. Désignée comme « impassible » (Schoots 16), cette œuvre procède d' " une tentative modeste de description du monde » (Bessard-Banquy 202 ; Jérusalem et Vray) tenant compte de la position du sujet contemporain dans celui-ci, tout en interrogeant la forme et l'action romanesque. Au-delà d'une "renarrativisation » caractéristique du récit postmoderne, elle crée, selon Christine Jérusalem, un « effet de romanesque » qui " vise l'adhésion du lecteur à l'aspect invraisemblable du récit et pas seulement à son aspect fictif (faux/vrai)» («Stevenson/Echenoz»332). En prenant Je m'en vais pour objet d'étude, je souhaite analyser la manière dont cette œuvre interroge l'indifférence actuelle qui existe envers un ailleurs rabattu sur du semblable ou réduit à un exotisme kitsch. Je fais l'hypothèse que, dans ce roman, Echenoz joue de la représentation pour questionner, tout en ayant recours à des images convenues, la simplification actuelle du lointain et renouvelle, par conséquent, le regard que nous y posons. De même qu'il n'est plus possible, à notre époque, d'aborder naïvement l'écriture romanesque pour cet auteur (Bessard-Banquy 194), l'autre et l'ailleurs ne peuvent davantage y faire l'objet d'une " compréhension parfaite d'un hors soi-même qu'on étreindrait en soi » (Segalen 44). Le dispositif 
du roman et son processus d'écriture suggèrent d'ailleurs que les représentations du lointain gagnent à conserver une certaine distance vis-à-vis de leur objet. Aussi, l'ironie dont la narration échenozienne fait preuve, en exhibant le dispositif symbolique, permettrait-elle de réirriguer un champ imaginaire dépourvu du manque qui supporte toute représentation (Piret 343).

Lauréat du prix Goncourt en 1999, Je m'en vais est un roman publié aux Éditions de Minuit, comme toutes les œuvres d'Echenoz, dont le titre fait explicitement référence au voyage ou, du moins, au déplacement. Il débute et se termine d'ailleurs avec ces mots. "Je m'en vais, dit Ferrer, je te quitte » (Echenoz 7), annonce Félix à sa femme Suzanne sur le seuil de leur domicile, en incipit du roman. " Je prends juste un verre et je m'en vais » (226), affirme le même Félix à une inconnue rencontrée dans une fête un an plus tard au même endroit - le seuil de son ancienne maison -, à la toute fin du roman. Entre ces deux moments, le protagoniste, marchand d'art propriétaire d'une galerie à Paris, vit diverses aventures dont une expédition dans l'Arctique canadien visant à récupérer un trésor oublié d'art inuit ancien. À cette occasion, il est confronté à une version désenchantée du Grand Nord dont Echenoz a voulu rendre « le blanc, le froid, un exotisme inversé » (Harang n.p.) alors que, encore aujourd'hui, l'exotisme est « volontiers "tropical” » (Segalen 33).

Ce n'est donc pas en touriste que Felix Ferrer aborde son voyage au départ de Paris, d'où il prend un avion pour Montréal, un autocar pour Québec, un briseglace pour Wager Bay au Nunavut, un plus petit avion pour Port Radium aux Territoires du Nord-Ouest, un traineau à chiens et des motoneiges pour se rendre à l'épave de la Nechilik, petit bateau de commerce prisonnier des glaces dans le golfe d'Amundsen depuis 1957 et contenant des objets d'art paléo-baleinien. La lassitude du personnage, partagée par la plupart de ceux qui peuplent l'œuvre d'Echenoz et à laquelle ont été consacrées plusieurs études (Blanckeman, À courir »; Jérusalem, « Petite étude »; Dangy), affermit la description ironique du désert glacé et de sa culture. Venu pour affaires et devant s'adapter au rythme ralenti par le froid et la géographie de ces latitudes, Ferrer «s'ennu[ie] sec» (Echenoz 88). Une fois récupérés les objets de l'épave et de retour à Port Radium, le personnage doit attendre qu'un artisan fabrique des contenants pour les transporter, sans que la ville ne lui offre beaucoup de divertissements : «Port Radium peut vraiment n'être pas marrant du tout, il ne s'y passe pas grand-chose, spécialement le dimanche où s'enchevêtrent étroitement, à leur plus haut degré d'efficacité, l'ennui, le silence et le froid » (Echenoz 88).

\section{« Jours heureux à Port Radium » : rapprochement, comparaison}

Port Radium n'a toutefois rien à envier aux destinations de voyage plus habituelles, comparable à celles-ci par la promiscuité et l'alcool qu'elle propose finalement au protagoniste, invité à manger chez la famille Aputiarjuk peu avant son départ :

Ferrer comme les autres y allant de son toast, on but généreusement à la chasse au phoque, on but affectueusement à la santé des chasseurs de phoques, on but avec enthousiasme à la santé des phoques en général et bientôt, l'alcool exaltant les affects, voici qu'on l'invitait même à passer la 
nuit là s'il le souhaitait, il partagerait sans aucun problème la chambre de la fille et l'on se raconterait ses rêves le lendemain comme ont coutume de faire, sous ces climats, toutes les familles tous les matins (Echenoz 91)

Tout en reconduisant certains stéréotypes des destinations de voyage - sexe, alcool, coutumes locales -, ce passage marque un écart vis-à-vis d'elles en les transposant, avec ironie, dans un climat inhospitalier, et sa chasse au phoque. Le rythme de la phrase, avec son accumulation en trois termes marquant la progression de l'ivresse, sa proposition à double sens faite au personnage de passer la nuit chez ses hôtes, et sa mention à la culture locale autour du rêve, appuie l'apparente banalité de la situation décrite. Sa répétition finale («toutes les familles tous les matins») couronne l'explication détachée du narrateur jouant sur la réception équivoque d'une histoire qui signifie doublement, en renvoyant à l'ébriété et à la chasse, qu'elle n'a pas pour vocation de présenter la vérité. En interrogeant, par sa description ironique, la vraisemblance d'une telle situation, le texte suscite dans un même temps une réflexion sur le statut des représentations de l'ailleurs, fiction aussi souple que peut l'être l'imagination, mise en évidence ici par la manière désinvolte dont la narration présente les choses. D'autant que la phrase suivante, qui clôt le chapitre, accumule les avantages de cette soirée polaire et se termine sur une hyperbole qui convaincrait peut-être le lecteur si elle ne contenait pas aussi une référence paradoxale à ce lieu qui évoque, finalement, la radioactivité et l'industrie minière : «Ferrer eut beaucoup de mal à refuser, les lampes donnaient une lumière douce et le poste diffusait du Tony Bennett, il faisait chaud, le poêle ronflait, tout le monde rigolait, la jeune fille lui souriait, ah, parlez-moi de Port Radium » (91). Difficile de prendre le narrateur au sérieux dans ses éloges qui, même s'ils peuvent déstabiliser les a priori sur l'Arctique en allant contre le sens commun, insistent sur leur artificialité par le retournement qu'ils marquent vis-à-vis du contexte par ailleurs inhospitalier et de l'ennui total précédemment suscité chez le protagoniste.

Le roman entretient une distante familiarité avec le Grand Nord qui passe largement par la modernité comparable de cette région, rapprochée de celle de l'Europe en ce qui concerne la maison de la famille Aputiarjuk, que le protagoniste découvre avant le repas : " on lui fit visiter la maison: bien isolée, téléphone et télévision, gros poêle et cuisine moderne, mobilier de bois blanc bon marché de genre nordique mais qu'on trouve jusqu'en banlieue parisienne » (90). Ainsi, le " genre nordique » du mobilier est à l'évidence construit par la commercialisation (et en Chine) et sa diffusion mondiale uniformise les intérieurs. La maison de Port Radium n'offre donc pas davantage de dépaysement qu'une autre en banlieue parisienne, rendant partiellement caduc l'intérêt pour la découverte que pouvait proposer le voyage. En marquant la ressemblance entre la décoration intérieure inuite et française, ce passage souligne aussi l'écart qui persiste entre les stéréotypes nordiques traditionnels ${ }^{1}$ et la réalité des communautés actuelles. En effet, la narration déconstruit certains clichés du monde polaire en mêlant tradition et

\footnotetext{
${ }^{1}$ Pensons notamment à ceux, toujours vivaces, véhiculés par le film Nanook of the North (1922) de Robert J. Flaherty, qui reçût une importante attention en France. Pensons notamment à ceux, toujours vivaces, véhiculés par le film Nanook of the North (1922) de Robert J. Flaherty, qui reçût une importante attention en France.
} 
technologie : les guides de Ferrer, par exemple, sont « vêtus d'édredon matelassé, de fibre polaire en synchilla, de sous-vêtements respirants en capilène, de combinaisons fluorescentes et de gants équipés d'un système chauffant » (49). La description détaillée de ces objets, dans un lexique spécialisé, a pour effet de gommer certaines différences entre ce monde et le pays d'origine du personnage, comme si les similitudes technologiques suffisaient pour créer une véritable communauté entre le voyageur et le pays d'accueil. Le roman présente toutefois une forme de comparatisme qui insiste sur la simplification de l'ailleurs et les limites que celle-ci impose à l'imaginaire de la différence. La France, la représentation d'une certaine France, est donnée comme unique point de repère culturel, freinant tout décentrement de la pensée, comme dans l'exemple culinaire que reprend l'intitulé de cet article et qui fait référence à l'adage « Tout est bon dans le cochon $»$ :

Or on sait que tout est bon dans le phoque, c'est un peu l'équivalent polaire du porc : sa chair se grille, se poche, se mijote, son sang au goût de blanc d'œuf donne un boudin correct, sa graisse permet de s'éclairer et de se chauffer, on fait de sa peau d'excellentes toiles de tente, ses os donnent des aiguilles et ses tendons du fil, on fabrique même avec ses intestins de jolis voilages transparents pour la maison (Echenoz 62)

Si «l'exotisme véritable», que Victor Segalen décrit comme « la notion du différent ; la perception du Divers ; la connaissance que quelque chose n'est pas soi-même » (41), est fondé sur une oscillation entre la reconnaissance de l'autre et le retour à soi (Baudrillard et Guillaume 53), on peut douter que cette forme de comparaison témoigne d'un tel exotisme, puisqu'elle implique que la découverte de l'ailleurs soit ramenée au connu plutôt que d'être appréciée en elle-même. Grâce à une accumulation qui rappelle l'utilisation versatile du phoque chez les Inuits, Echenoz dénonce l'assimilation qui peut en être faite par une description ethnocentrée. Ainsi, le boudin de phoque est « correct» parce qu'il se conforme aux goûts et attentes implicites de la charcuterie française. Tandis que l'adjectif «jolis » contraste avec le registre tripier des intestins dans l'élément final de l'énumération, suggérant d'abord une appréciation différente de la matière, associé aux «voilages transparents pour la maison», il tire néanmoins badinement la comparaison vers une forme de décoration intérieure occidentale. L'ironie du narrateur montre ici qu'il n'est pas dupe de cette appropriation de spécificités locales, comblant l'écart entre l'ici et l'ailleurs, le même et l'autre, à l'instar de la marchandisation culturelle qui rend «accessibles » des traditions lointaines. Dans Je m'en vais, cette apparente et paradoxale proximité, relevant souvent de l'anecdote, est donc mise en parallèle avec une véritable étrangeté au monde polaire. Or, cette dynamique de simultanéité s'inscrit dans une esthétique postmoderne, dans laquelle les stéréotypes « sont tour à tour légitimés et dénoncés, utilisés et déconstruits » (Dufays 87). L'effet de romanesque qui guide l'écriture d'Echenoz n'a donc rien d'innocent et engage le lecteur à la fois à « se moquer et se délecter des conventions artificielles » (Jérusalem, « Stevenson/Echenoz » 335).

\section{« Très loin, très blanc, très froid " : perception, représentation}


Cette rencontre mitigée avec le Grand Nord, entre assimilation et superficialité, peut toutefois s'expliquer par la difficulté de saisir un tel endroit, qui déjoue la perception occidentale de l'espace. Lorsque Ferrer est d'abord informé de l'emplacement de l'épave, il consulte son atlas afin de s'en faire une idée. Or,

Les pôles, chacun peut l'éprouver, sont les régions du monde les plus difficiles à regarder sur une carte. On n'y trouve jamais bien son compte. De deux choses l'une en effet. On peut d'abord essayer de les considérer comme occupant le haut et le bas d'un planisphère classique, l'équateur étant pris comme basse horizontale médiane. Mais dans ces conditions tout se passe comme si on les regardait de profil, en perspective fugitive et toujours forcément incomplets, ce n'est pas satisfaisant. Ensuite on peut aussi les regarder par en dessus, comme vus d'avion: de telles cartes existent. Mais alors c'est à leur articulation avec les continents, qu'habituellement on voit pour ainsi dire de face, que l'on ne comprend plus rien et ça ne va pas non plus. Ainsi les pôles sont-ils rétifs à l'espace plat. Obligeant à penser en plusieurs dimensions en même temps, ils posent un maximum de problèmes à l'intelligence cartographique. Mieux vaudrait disposer d'un globe terrestre, or Ferrer n'en a pas. Mais bon, il parvient quand même à se faire une petite idée du coin : très loin, très blanc, très froid (Echenoz 68)

Ce passage montre bien les difficultés cartographiques, toujours réelles à notre époque pourtant riche en imagerie satellite, de (se) représenter un lieu situé aux antipodes de la terre. De plus, les deux possibilités insatisfaisantes de visualisation évoquées par le narrateur semblent faire pendant aux deux risques, évoqués par Baudrillard et Guillaume, que présente la rencontre avec l'ailleurs : le planisphère classique accommode la vision occidentale et permet de se situer vis-à-vis de l'Arctique sans toutefois en avoir une image complète, tandis que la projection polaire montre bien l'Arctique, mais rend son positionnement par rapport aux continents malaisé pour un habitué de la projection de Mercator. Dans les deux cas, le réel défie «l'intelligence cartographique » qui, on s'en aperçoit à la personnification des pôles (« rétifs à l'espace plat»), n'a rien d'intelligent, mais tout de l'arbitraire de la représentation. En effet, comme le rappelle Gilles Tiberghien citant Peter Sloterdijk,

Par leur nom comme sur le fond, les planisphères - littéralement les boules plates - veulent éliminer le souvenir de la troisième dimension non dominée par la représentation .... Quand on réduit la profondeur, on met la main sur le réel. ... Lorsqu'on réussit à capturer les sphères sur le papier et à simuler des profondeurs spatiales sur des toiles, des possibilités nouvelles et infinies s'ouvrent à la conquête du monde comme image. (111)

« La conquête du monde comme image », c'est bien ce dont il s'agit à notre époque tyrannisée par le visible (Aubert et Haroche), même si ce fantasme est illusoire et que le réel résiste toujours à la représentation. Toute l'ironie d'Echenoz vise précisément à rappeler cette résistance qui s'inscrit, en creux, dans la représentation même, et que les théoriciens de la littérature ont décrite de manières diverses: brutalité (Mathet Brutalité), incompréhensible (Mathet L'Incompréhensible), 
faillite (Rykner Paroles), « pan » (Rykner Pans), etc. Aussi, la « petite idée » que se fait le protagoniste du « coin » est-elle résolument réductrice, caricaturale, tout en témoignant de l'échec du langage et de l'imagination lorsqu'il s'agit de décrire un lieu aussi étranger pour un Parisien que celui de la banquise.

Même lorsque le personnage s'y rend, le lieu se dérobe et rappelle les déserts chauds et leurs mirages, de sorte qu'il conserve toujours une part d'indéfinissable :

L'illusion règne en effet sous ces climats. La veille encore, tenez, on avançait derrière ses lunettes noires, sans lesquelles le soleil arctique vous emplit les yeux de sable et la tête de plomb, quand ce même soleil s'était soudain multiplié dans les nuages glacés par effet de parhélie. Ferrer et ses guides s'étaient retrouvés aveuglés par cinq soleils simultanément, horizontalement alignés, parmi lesquels le vrai - avec deux autres astres supplémentaires à la verticale du vrai. Ç'avait duré une petite heure avant que ce vrai soleil se retrouvât tout seul (Echenoz 73)

Bien que le parhélie soit un phénomène optique qui se produit dans une atmosphère chargée de cristaux de glace présents dans les nuages de haute altitude et donc plus courant dans les régions polaires, le champ lexical de la description (lunettes noires, sable, plomb, soleil) rappelle paradoxalement les déserts chauds. La présence de « faux soleils » côtoyant le vrai souligne la part d'illusion que comporte notre perception et, dès lors, l'incertitude qu'elle peut engendrer. Elle rappelle, dans un même temps, la contingence de la représentation dans laquelle elle s'inscrit et sa facilité à reprendre des motifs connus du récit de voyage, mais aussi de les détourner pour renouveler l'imaginaire du Nord.

\section{« Je m'en vais » : déplacement, fuite}

Tout en conservant une part d'insaisissable, l'ailleurs polaire ne semble pas être l'occasion, pour le protagoniste de Je m'en vais, de découvrir et de s'enrichir, ne serait-ce que pour mieux se (re)trouver au retour. Félix Ferrer semble, au contraire, souhaiter se délester de lui-même par divers moyens, allant de la distraction au sommeil. Comme l'annonce le titre du roman, l'intérêt du voyage est pour lui, comme pour les autres personnages échenoziens, "purement vectoriel, c'est-à-dire qu'il s'agit simplement d'une question de déplacement, ... de circulation » (Baudrillard et Guillaume 73). Dans ce type de voyage, qualifié de contemporain par Jean Baudrillard, « le rythme n'est plus la découverte, l'échange, mais c'est une sorte de déterritorialisation en douceur, une sorte de séduction par l'absence » (74). Pour le philosophe, "vous êtes pris en charge par le voyage luimême, donc par l'absence, qui est une absence à vous-mêmes également. À ce moment-là, vous êtes dans un avion, vous n'êtes plus responsable de rien » (74). Cette description du voyage contemporain rappelle les «joies passives » (Augé 129) que Marc Augé associe aux déplacements à travers les non-lieux, dont l'aéroport et l'avion sont des archétypes, et qui sont l'occasion, dans le roman, de souligner que « les personnages d'Echenoz ne cessent de fuir le vide qui s'ouvre sous eux, en eux, et dont l'ennui est le signe » (Blanckeman, «À courir » 130). En effet, le trajet de Ferrer entre Paris et Montréal montre le désintérêt du personnage 
pour lui-même et pour l'autre à travers le voyage, dont le rythme accumule les lieux sans qu'ils ne semblent prendre une signification particulière pour lui, signalant une " géographie du vide» minutieusement décrite par Christine Jérusalem (Jean Echenoz). À bord de l'avion, par exemple, il va d'abord «distinguer une étendue de mer, ornée d'une île qu'il ne pourra identifier, puis une étendue de terre au cœur de laquelle c'est un lac, cette fois, dont il ne connaîtra pas le nom » (Echenoz 12). Son indifférence pour le paysage est par ailleurs équivalente à celle qu'il affiche pour l'intérieur de la cabine, dans laquelle « il somnole », « il suit nonchalamment sur un écran quelques pré-génériques de films », il est "distrait par les allées et venues des hôtesses qui ne sont peut-être plus ce qu'elles ont été » (12). Le déplacement du personnage est donc source d'ennui, spécifiquement associé à une époque distincte de celle, révolue, où les agentes de bord étaient associées à un cliché de séduction en uniforme, marquant à la fois la temporalité contemporaine et son esthétique, romanesque, de détournement des stéréotypes. Devenu espace de transit plutôt qu'aventure futuriste de la modernité, l'avion crée des solitudes, susceptibles d'entraîner des rêveries personnelles, sans toutefois qu'elles ne soient bien accueillies par le voyageur :

À deux cents compressés dans une carlingue, on est en effet isolé comme jamais. Cette solitude passive, pense-t-on, serait peut-être l'occasion de faire le point sur sa vie, de réfléchir au sens des choses qui la produisent. On essaie un moment, on se force un peu mais on n'insiste pas longtemps devant le monologue intérieur décousu qui en résulte et donc on laisse tomber, on se pelotonne et s'engourdit, on aimerait bien dormir, on demande un verre à l'hôtesse car on n'en dormira que mieux, puis on lui demande un autre pour faire passer le comprimé hypnotique : on dort (Echenoz 12)

L'introspection ratée du personnage et son recours à un somnifère, présentés comme récurrents et généralisés aux autres passagers par l'usage du temps présent et du pronom « on ", suggèrent la dénonciation d'un malaise existentiel du sujet contemporain lié à l'isolement, pourtant simultané à la grande proximité qui existe dans un avion, et à un besoin de disparition, au moins temporaire. "Tentation contemporaine » (Le Breton) selon David Le Breton, cette absence à soi déclinée dans l'œuvre d'Echenoz pourrait expliquer la rencontre mitigée du personnage avec l'autre et l'ailleurs et, dès lors, la représentation équivoque qui en est faite. La surabondance spatiale ne serait donc pas l'unique cause de la banalisation actuelle $\mathrm{du}$ lointain et de l'assèchement de son imaginaire; le malaise du sujet contemporain, décrit par la psychanalyse (Lebrun; Dufour; Melman) et touchant à la dimension du désir et à l'ordre symbolique, contribuerait également à limiter la possibilité du dépaysement. Par ailleurs, la rencontre ratée du personnage avec le Nord canadien montre que la présence n'est pas gage de découverte authentique de l'ailleurs, contrairement à ce que laisse imaginer une telle expédition.

\section{distance \\ « Cette opération vers le nord restait à l'état d'hypothèse » : présence,}

Contrairement aux récits de voyages et au rapport plus étroit qu'ils nouent avec la vérité géographique, on octroie davantage de liberté aux romans qui 
dépeignent des lieux réels, tout en s'interrogeant à l'occasion sur l'exactitude de leurs descriptions. L'œuvre d'Echenoz, caractérisée par l'accumulation de termes techniques et scientifiques, donne souvent l'impression d'être particulièrement documentée, s'attachant minutieusement aux détails, dans « un souci d'observation topographique et de consignation matérielle » (Blanckeman, "En théorie » 81). Ces détails sont effectivement vrais, comme l'auteur le précise à propos de Je m'en vais :

Tout ce qu'il y a dans ce livre est exact, les caractéristiques de la Nechilik (un bateau de commerce long de 23 mètres, construit en 1942 et enregistré à Saint John au New Brunswick), les coordonnées de son échouage, celles du brise-glace Le Desgroseillers (13600 chevaux, 7,16 mètres de tirant d'eau) mais également toutes les œuvres d'art inuit existent réellement (elles n'ont évidemment pas été retrouvées dans la Nechilik, où ne restaient que deux lampes à pétrole que je cite), tout ce qui se passe dans les hôpitaux (je n'ai pas mon pareil pour distinguer un B.A.V., bloc auriculo-ventriculaire, de type Mobitz d'un Luchiani-Wenckerbach), dans les tribunaux ou les galeries, est vrai. Je me documente beaucoup, je rencontre des gens, je lis, je compile toute sorte de catalogues, je repère les noms des photographes, je vais les voir, j'enregistre, je retape tout ce que j'écris (Harang n.p.)

Malgré leur exactitude, ces détails jouent plutôt en faveur d'un effet de romanesque selon Christine Jérusalem ( « Stevenson/Echenoz » 337), leur sélection servant la fiction avant tout, comme le suggère d'ailleurs la démarche d'Echenoz vis-à-vis de la documentation qu'il accumule dans ses projets d'écriture : "Attention, ce n'est pas pour utiliser ces renseignements, c'est une sorte de garde-fou, j'en garde peutêtre $1 \%$, il n'y a jamais plus d' $1 \%$ de la réalité qui soit pertinent en terme de romanesque, mais ces détails-là sont toujours plus romanesques que ceux que l'on pourrait inventer » (Harang). Ainsi, les détails géographiques qui donnent vie à la représentation de lieux étrangers, même s'ils s'appuient sur la réalité, produisent un monde fictionnel où règnent aussi l'invraisemblable, l'extraordinaire, l'artifice.

L'écriture échenozienne rappelle que les représentations de l'ailleurs ne reposent pas uniquement sur des faits et le voyage sur des observations, mais qu'elles constituent aussi une aventure imaginaire, intérieure, ressentie. La présence psychique serait donc plus importante que la présence physique pour saisir les lieux lointains et ce, en dépit de la facilité à notre époque, de les visiter. Le voyage indirect que permet la lecture, dans les mondes construits de la littérature, insiste, par la sélection narrative qu'il opère, sur la «mise en sens du réel, qui contribue à le découper et à l'organiser » (Bayard 38). Il concourt ainsi à déconstruire l'idée qu'il existe une manière objective et authentique d'appréhender un lieu, sans qu'il ne soit affecté par un ensemble de projections, de fantasmes, d'images, de perceptions, etc. La distance littéraire et les lois auxquelles elle soumet les lieux qu'elle décrit sont d'ailleurs inscrites dans la trame narrative de Je m'en vais, dont l'expédition polaire est déléguée à Félix Ferrer par son employé, Delahaye/Baumgartner, qui vole ensuite le trésor inuit rapporté à Paris sans avoir eu à se rendre lui-même dans l'Arctique. Le roman repose donc en quelque sorte sur l'évitement du voyage, considéré comme « perdu » et « pur déficit » (Echenoz 138) par Ferrer dès lors que les antiquités lui sont dérobées. Son aventure nordique 
est également tenue responsable, quelques jours après son retour à Paris dans la chaleur de juillet, de lui provoquer un arrêt cardiaque. Le personnage, souffrant de troubles coronariens, s'est en effet exposé à des variations de température importantes contre les recommandations de son cardiologue : «Tu évites de t'exposer à des températures extrêmes, hein, pas trop de froid ni trop de chaud parce que, je te l'ai dit, c'est une chose catastrophique pour les gens comme toi » (Echenoz 117). La découverte du Nord canadien n'est donc pas présentée de manière positive par le roman, dont le lecteur semble être, indirectement, le seul bénéficiaire. Celui-ci peut ainsi expérimenter le monde polaire malgré le « deuil de la fascination que put longtemps exercer l'ailleurs » (Carlat 73) que Dominique Carlat associe aux Grandes blondes et qui teinte en réalité toute l'œuvre voyageuse d'Echenoz. Cette appréciation paradoxale du lecteur est rendue possible par le traitement ironique de l'auteur, qui met à distance l'exotisme réduit à des images de pacotille tout en permettant au lecteur d'entrer dans le monde du récit : « l'ironie représente en cela un mode d'énonciation privilégié, parce qu'elle interroge les figures de la fiction sans en interdire l'effet » (Blanckeman, « En théorie » 79). En effet, la position critique du narrateur échenozien est uniquement suggérée, laissant une place à la bivalence du stéréotype, de sorte que son " sort interprétatif [...] est incertain, soumis à l'appréciation du récepteur » (Dufays 81).

Le souci de produire une expérience affective de l'ailleurs chez le lecteur se trouve dans la démarche même d'Echenoz, qui se documente énormément, mais toujours dans le but de servir la fiction. Bien qu'il fasse régulièrement du repérage pour écrire ses romans, à Paris et ailleurs, il a préféré ne pas se rendre dans le Grand Nord pour écrire Je m'en vais:

Et je ne voulais pas aller sur place : pas seulement parce que je suis frileux, mais surtout parce que $\mathrm{j}$ 'avais le souvenir que le travail de documentation que j'avais fait en Inde [pour Les Grandes blondes], s'il avait été finalement très précieux parce que j'avais pris beaucoup de notes, avait aussi un peu restreint le champ de la fiction, de l'imagination, de l'aventure, comme si j'avais été trop encadré par la réalité... Pour le Grand Nord, j'ai donc choisi de me documenter sans y aller (Echenoz 232)

En montrant le besoin de maintenir une certaine distance vis-à-vis de son objet, l'auteur fait écho à la nécessité conserver un « en dehors » à soi (Segalen 38) dans l'appréhension de l'ailleurs, mais également dans la fiction, qui permet d'ouvrir l'imaginaire, de voir les choses différemment. En insistant sur la part de fiction, de mise en récit toujours indispensable dans la construction du lointain à notre époque, Echenoz marque la spécificité de la littérature par rapport aux autres moyens de description du monde. L'ironie qu'il utilise dans ses romans place le lecteur au centre de ces préoccupations, lui laissant la liberté de développer sa propre vision critique : «Conscient de la superficialité de l'univers qu'il met en scène, le narrateur refuse d'endosser le rôle de censeur ; l'élégance de son propos, hautement revendiquée, tient à la légèreté qu'il entretient. Cette dernière ne peut souffrir d'une position de surplomb trop pesamment investie » (Carlat 73). Ainsi, c'est toujours dans l'écart que se construisent les représentations de l'ailleurs, dont la distance géographique illustre la distance que comporte toute représentation, de laquelle 
l'esthétique postmoderne d'Echenoz se joue en créant « une réalité qui a l'air d'un simulacre » (Jérusalem, « Stevenson/Echenoz » 337).

\section{Conclusion}

L'ailleurs contemporain, même s'il se construit en tenant compte des possibilités techniques qu'offre notre époque et des rapprochements qu'elles permettent, ne peut faire complètement abstraction des différences qui l'opposent à l'ici. L'affaiblissement de l'exotisme géographique dû à l'accroissement de la circulation vers les pays lointains entretient une apparente proximité des peuples et des lieux qui se traduit plus par une production de stéréotypes qu'une réelle (re)connaissance de l'autre. Dans Je m'en vais, Jean Echenoz fait ressortir cette simplification de l'ailleurs dont souffre notre monde tout en insistant sur le possible exotisme qu'il peut toujours présenter, le plus souvent là où il n'est pas attendu. L'auteur réussit, par le biais de certains stéréotypes du voyage, à détourner le regard habituellement posé sur les lieux lointains et à désactiver les poncifs qui courent à leur sujet. Ce faisant, il réaménage une distance vis-à-vis d'eux sans compromettre l'agrément du lecteur, dont la lecture oscille entre le plaisir de la célébration des stéréotypes et celui de leur mise à distance par l'ironie (Dufays 84), qui invite à poser deux regards contradictoires sur les topoi de l'ailleurs (Dufays 87) : un regard de reconnaissance des images connues de l'Arctique et de ses habitants et, en même temps, un regard lucide sur ces constructions tournées en dérision par l'auteur. Cette forme de distante familiarité expérimentée par rapport à l'ailleurs polaire rend compte de l'apparente uniformisation actuelle de la planète, mais aussi du positionnement de chacun vis-à-vis de l'autre et de son milieu.

Nous avons pu constater que les représentations du Nord canadien dans Je m'en vais jouent sur leurs similitudes avec celles de la France, dans un impossible décentrement. Si elles participent à défaire les clichés qui ont cours sur l'Arctique, elles rappellent aussi la part d'artificialité que comporte toute représentation, qui est toujours représentation de l'absence. La difficulté, pour le personnage principal, de saisir ce lieu qui, même lorsqu'il l'explore, se révèle trompeur, souligne le manque structurel de la représentation et, par conséquent, réaménage un espace pour l'imaginaire, hors des lieux communs. Même si les déplacements du personnage témoignent de la facilité, aujourd'hui, de se rendre dans des lieux lointains et isolés, sa rencontre ratée avec ceux-ci insiste sur l'angoisse et l'ennui contemporains et la difficulté toujours réelle d'avoir accès à l'ailleurs. À rebours de la découverte et du choc que peut offrir le voyage, et de l'opportunité introspective qu'il présente, les déplacements de Félix Ferrer présentent son désintérêt pour les contrées qu'il sillonne et sa motivation financière pour le trésor inuit. Son absence aux lieux où il se trouve va de pair avec son absence à lui-même, motif récurrent chez Echenoz et témoin, selon Bruno Blanckeman, de notre époque: "Perte de consistance, subjectivité problématique, individualité contrefaite : la fiction transpose, par ses moyens, une peine à être qu'elle propose comme empreinte des temps présents » (Les Récits indécidables 70). Les romans géographiques d'Echenoz, avec leur lot de rencontres et de découvertes souvent mitigées, sont particulièrement bien placés pour signaler les malaises du sujet 
contemporain, tout en tentant « de désubstantialiser la représentation, d'en révéler l'envers et le défaut » (Piret 343).

En faisant la promotion du non-voyage, Je m'en vais minimise la nécessité d'une présence physique pour faire l'expérience de l'ailleurs et souligne la part de subjectivité qui entre dans celle-ci, déterminée dans tous les cas par notre manière de saisir le réel. La présence psychique au lointain qu'offre le récit, par l'intermédiaire des descriptions qu'il en fait, transporte le lecteur tout en marquant la distance intrinsèque à toute appréhension de l'ailleurs. Ainsi, la fiction échenozienne et l'effet de romanesque qu'elle crée auprès des lecteurs permettent un certain réenchantement du monde par le biais d'une présence indirecte, à rebours de l'exigence du visible actuelle, rappelant la vivacité de la réalité psychique et l'incertitude de la limite entre voyage et non-voyage, « liée avec la part de fiction qui accompagne toute description d'un lieu » (Bayard 29).

\section{Bibliographie}

Aubert, Nicole et Claudine Haroche (dir.). Les Tyrannies de la visibilité : être visible pour exister? Ramonville : Érès, 2011. Imprimé.

Augé, Marc. Non-lieux : introduction à une anthropologie de la surmodernité. Paris : Seuil, 1992. Imprimé.

Baudrillard, Jean et Marc Guillaume. Figures de l'altérité. Paris : Descartes et Cie, 1992. Imprimé.

Bayard, Pierre. Comment parler des lieux où l'on n'a pas été ? Paris : Minuit, 2012. Imprimé.

Bessard-Banquy, Olivier. " Il se passe quelque chose avec le jazz. Entretien avec Jean Echenoz. » Europe 75 (1997) : 194-202. Imprimé. 
Blanckeman, Bruno. "À courir pour courir. », Siècle 21. Littératures et société 17 (2010) : 124-131. Imprimé.

---. Les Récits indécidables: Jean Echenoz, Hervé Guibert, Pascal Quignard. Villeneuve d'Ascq : Presses universitaires du Septentrion, 2008. Imprimé.

---. « En théorie, “je m'en vais". » Roman 20-50 38 (2004) : 77-83. Imprimé.

Carlat, Dominique. «De la vertu critique de l'humour à l'ère industrielle de la reproduction: Les Grandes blondes de Jean Echenoz. » Roman 20-50 38 (2004) : 71-76. Imprimé.

Dangy, Isabelle. « Suspension, détachement, apesanteur dans les romans de Jean Echenoz. ", Romanciers minimalistes 1979-2003. Blanckeman, Bruno et Marc Dambre (dir.). Paris : Presses Sorbonne Nouvelle, 2012. 177-185. Imprimé.

Dufays, Jean-Louis. "Stéréotypes, lecture littéraire et postmodernisme. », Lieux communs, topoï, stéréotypes, clichés. Plantin, Christian (dir.). Paris : Kimé, 1993. 80-91. Imprimé.

Dufour, Dany-Robert. L'Art de réduire les têtes : sur la nouvelle servitude de l'homme libéré à l'ère du capitalisme total. Paris : Denoël, 2003. Imprimé.

Echenoz, Jean. Je m'en vais. Paris : Minuit, 2001 [1999]. Imprimé.

Harang, Jean-Baptiste. « La réalité en fait trop, il faut la calmer. Entretien avec Jean Echenoz. » next.liberation.fr. Libération, 16 septembre 1999. Web. 04/07/2016.

Jérusalem, Christine. «Petite étude de la mélancolie échenozienne. » Siècle 21. Littératures et société 17 (2010) : 145-156. Imprimé.

---. Jean Echenoz: géographies du vide. Saint-Étienne: Publications de l'Université de Saint-Étienne, 2005. Imprimé.

---. «Stevenson/Echenoz: le jeu des "images irréelles". » Le Roman français au tournant du XXI $I^{\text {eme }}$ siècle. Blanckeman, Bruno, Aline Mura-Brunel et Marc Dambre (dir.). Paris : Presses Sorbonne Nouvelle, 2004. 331-339. Imprimé.

Jérusalem, Christine et Jean-Bernard Vray (dir.). Jean Echenoz : " une tentative modeste de description du monde ». Saint-Étienne: Publications de l’Université de Saint-Étienne, 2006. Imprimé.

Le Breton, David. Disparaître de soi: une tentation contemporaine. Paris: Métailié, 2015. Imprimé.

Lebrun, Jean-Pierre. Un monde sans limite: essai pour une clinique psychanalytique du social. Ramonville : Érès, 2003. Imprimé.

Mathet, Marie-Thérèse (dir.). Brutalité et représentation. Paris : L'Harmattan, 2006. Imprimé.

---. L'Incompréhensible: littérature, réel, visuel. Paris: L'Harmattan, 2003. Imprimé.

Melman, Charles. La Nouvelle économie psychique : la façon de penser et de jouir aujourd 'hui. Ramonville : Érès, 2009. Imprimé.

Piret, Pierre. «Le dispositif minimaliste et la dialectique du désir (Echenoz, Toussaint). » Représenter à l'époque contemporaine : pratiques littéraires, artistiques et philosophiques. Ost, Isabelle, Pierre Piret et Laurent Van Eynde (dir.). Bruxelles : Facultés universitaires Saint-Louis, 2010. 325-343. Imprimé. 
Rykner, Arnaud. Pans : liberté de l'œuvre et résistance du texte. Paris : José Corti, 2004. Imprimé.

---. Paroles perdues : faillite du langage et représentation. Paris : José Corti, 2000. Imprimé.

Segalen, Victor. Essai sur l'exotisme : une esthétique du divers. Paris : Le livre de poche, 2014 [Fata Morgana, 1978]. Imprimé.

Schoots, Fieke. « Passer en douce à la douane ». L'écriture minimaliste de Minuit : Deville, Echenoz, Redonnet et Toussaint. Amsterdam : Rodopi, 1997. Imprimé.

Tiberghien, Gilles. Finis Terrae: imaginaires et imaginations cartographiques. Paris : Bayard, 2007. Imprimé. 